\title{
EFECTOS DE UNA SESIÓN DE ENTRENAMIENTO CONCURRENTE SOBRE LA PRECISIÓN Y EFECTIVIDAD EN EL TIRO DEPORTIVO CON PISTOLA DE AIRE $10 \mathrm{M}^{1}$ EFECTS OF A CONCURRENT TRAINING SESSION ON THE PRECISION AND EFFECTIVENSS IN SHOOTING SPORT $10 \mathrm{M}$ WITH AIR GUN
}

Cómo citar: Hernández, C., Aroca, J., Herreño, E., \& Gutiérrez, C. (2019). Efectos de una sesión de entrenamiento concurrente sobre la precisión y efectividad en el tiro deportivo con pistola de aire $10 \mathrm{~m}$. R. Actividad fis. y deporte. 6 (1): 58-67.

Artículo de acceso abierto publicado por: Revista Digital: Actividad Física y Deporte, bajo una licencia Creative Commons CC BY-NC 4.0.

\section{Hernández Uribe Carlos Daniel}

Profesional en Educación Física Militar, Subteniente del Ejército Nacional de Colombia.

E-mail: carlito_159@hotmail.com

\section{Aroca Gómez Juan David}

Profesional en Educación Física Militar, Subteniente del Ejército Nacional de Colombia.

E-mail: juandavid971002@outlook.com

\section{Herreño Larrota Edwin Farith Jahir}

Profesional en Educación Física Militar, Subteniente del Ejército Nacional de Colombia.

E-mail: farithjahir_1996@hotmail.com

\section{MsC. Catalina Gutiérrez Gómez}

Profesional en Ciencias del Deporte, Magister en Ciencias del Deporte. Asesora de trabajo de grado de la Facultad Educación Física Militar.

E-mail: cata_gutierrez9@hotmail.com

\section{RESUMEN}

Introducción: la Formación Integral Militar requiere de una preparación constante de cuerpo y mente para el desarrollo de operaciones y actividades de alto riesgo que precisan de un alto nivel de entrenamiento. El Entrenamiento Concurrente es una fusión del desarrollo de la fuerza y la resistencia para integrar la aptitud física de los individuos, buscando una mejora de su capacidad cardiorrespiratoria y su capacidad contráctil. Objetivo: analizar el efecto de una sesión de entrenamiento empleando cargas de resistencia y fuerza en el mismo grupo, sobre la frecuencia cardíaca y verificar la calidad de la precisión en el tiro deportivo a $10 \mathrm{~m}$ en pistola de aire. Metodología: estudio cuasi-experimental, con una muestra a conveniencia, tomando registro de los Cadetes de la Compañía Sucre,

\footnotetext{
${ }^{1}$ Artículo presentado como documento final requisito para obtener el título como profesional en Educación Física Militar.
} 
siendo seleccionados los Cadetes del aula 5TE5 adscritos a la Facultad de Educación Física Militar $\mathrm{N}=20$. Resultados: se desarrolla una parametrización considerando una distribución normal de estos por medio de la prueba de Shapiro-Wilk a partir de la media aritmética. Conclusión: el entrenamiento de la fuerza genera un mayor cambio en la FC, por lo cual, la postura y la respiración no generan un control adecuado y afecta la precisión del disparo.

Palabras Clave: Formación Integral Militar, Entrenamiento Concurrente, Frecuencia Cardíaca, Tiro Deportivo, Precisión de Disparo.

\section{ABSTRACT}

Introduction: Comprehensive Military Training requires a constant preparation of body and mind for the development of highrisk operations and activities that require a high level of training. Concurrent Training is a fusion of the development of strength and endurance to integrate the physical fitness of individuals, seeking an improvement in their cardiorespiratory capacity and contractile capacity. Objetive: analyze the effect of a training session using resistance and force loads in the same group, on the heart rate and verify the quality of accuracy in the sports shot at 10 m by air gun. Methodology: quasiexperimental study, with a sample to convenience, taking record of the Cadets of the Sucre Company, being selected the
Cadets of the 5TE5 classroom assigned to the Faculty of Military Physical Education N = 20 . Results: a parameterization is developed considering a normal distribution of these by means of the Shapiro-Wilk test based on the arithmetic mean. Conclusion: Strength training generates a greater change in $\mathrm{HR}$, so that posture and breathing do not generate adequate control and affect the accuracy of the shot.

Key Words: Integral Military Training, Concurrent Training, Heart Rate, Shotting Sport, Quality of the Precision.

\section{INTRODUCCIÓN}

El desarrollo de actividades físico deportivas es un elemento que produce modificaciones en el funcionamiento del Sistema Cardiovascular (SCV) y sus mecanismos autonómicos. La Frecuencia Cardíaca es considerada como una de las señales vitales más importantes del organismo; siendo usada frecuentemente en los exámenes de diagnóstico médico, debido a que, su análisis en relación de sus ondas puede predecir señales de riesgo para la salud o adaptaciones causadas por el proceso de entrenamiento (Figueiredo \& De Barros, 2018).

De acuerdo Cristancho, Otálora, \& Callejas, (2016), la Frecuencia Cardíaca (FC) se define como las veces que se presenta el volumen de eyección sistólico en el corazón, por un período determinado de tiempo un (1) 
minuto y su función es generar el transporte de sangre con mayor concentración de oxígeno, minerales y glóbulos blancos a los diferentes órganos de acuerdo a las necesidades presentadas. Para Fuentes \& Díaz (2010), un referente del desarrollo del Sistema Nervioso Autónomo (SNA) es la Frecuencia Cardíaca en Reposo (FCRp) de una persona, la que debe estar entre 60 y 100 pulsación por minuto (ppm).

De esta forma, "el desarrollo de diferentes modalidades como el tiro deportivo, siendo este un deporte en el que se pone a prueba la precisión y la velocidad en el manejo de determinadas armas, que pueden ser de fuego o de aire comprimido" (Mano, 2010, p. 3) y su objetivo está relacionado con un blanco conocido como la diana. Sus inicios se relacionan como un medio de defensa y de cacería, para el desarrollo de una alta precisión en el momento del disparo. Esto ha ayudado a desarrollar acciones para el entrenamiento de la técnica. Especialmente, por la importancia en sus tareas en el ámbito militar por lo que el tirador debe tener una adecuada capacidad y control de la respiración, para el desempeño acorde de su técnica y el control de la táctica (Real Federación Española de Tiro Olímpico, 2007).

La correcta posición sobre la pistola y el control de la respiración permiten tener una mejor efectividad en el disparo, debido a que, en el desarrollo de las acciones militares, la precisión es de vital importancia; porque este es el principal mecanismo de combate para la defensa de los combatientes y de las poblaciones.

El uso del Tiro Deportivo como deporte en la modalidad de pistola en la prueba $20 \mathrm{~m}$, consiste en apuntar con el arma a un objetivo estático, por lo que sirve para generar procesos de entrenamiento reales con los cadetes de la Escuela Militar, ayudando al mejoramiento de sus destrezas a la hora de disparar. Así mismo, permite tener una mayor reacción sobre el blanco objetivo del disparo (Real Federación Española de Tiro Olímpico, 2007).

Uno de los principales referentes para generar un disparo preciso requiere de una adecuada postura, que se describe como una apertura de los pies a lo ancho de los hombros, en la inclinación del cuerpo, en el ajuste del codo para no generar movimientos, la rotación de la cadera para hacer equilibrio y en la fuerza del hombro para sostener el arma (Ministério da Justiça e Segurança Pública, 2012). Por último, la efectividad del disparo en la precisión, debe adquirir una adecuada técnica de respiración: 1.- antes de levantar el brazo, 2.- inspirar y 3.- expirar repetidamente, pero no tan profundamente para que se controlen las pulsaciones. En el desarrollo de la respiración hay un punto medio para que se ejecute el disparo en el momento adecuado para expirar, en ese tiempo se apunta y se dispara, porque no hay ningún movimiento de: 1.- la caja torácica, 2.el estómago y 3.- otra situación que no permita generar movimientos bruscos 
(Ministério da Justiça e Segurança Pública, 2012).

Este estudio tiene como finalidad el análisis de la frecuencia cardíaca en dos grupos de cadetes de la Facultad de Educación Física Militar (FEFM) que fueron entrenados con trabajos de fuerza (G1) y resistencia aeróbica (G2). Se compara la precisión de diez (10) disparos antes y después del proceso de entrenamiento.

\section{METODOLOGÍA}

Se desarrolló un estudio cuasiexperimental descriptivo de tipo cuantitativo de corte longitudinal. La muestra se selecciona a conveniencia, tomando registro de los Cadetes de la Compañía Sucre, siendo seleccionados los Cadetes del aula 5TE5 adscritos a la Facultad de Educación Física Militar $(\mathrm{N}=20)$ varones $\mathrm{n}=18$ y mujeres $\mathrm{n}=2$. Para la toma de muestras se establece el cumplimiento de la Resolución 8430 de 1993, en la que se establecen las normas para la investigación en salud en Colombia, este estudio se considera de riesgo mínimo y se solicita un consentimiento informado por escrito, manteniendo en confidencialidad el uso de los datos encontrados y la identidad de los estudiados (Ministerio de Salud y Protección Social, 1993).

Se excluyen los participantes que presentan incapacidad médica, lesión osteomuscular y otras patologías de acuerdo a certificado médico.

\section{PROCEDIMIENTO}

Se desarrolló la toma de datos con los Cadetes de la compañía Sucre de la Escuela Militar José María Córdova, pertenecientes a la Facultad de Educación Física Militar, que cumplen con criterios de inclusión.

Se emplean monitores de FC Polar FT60 para el registro de la FC, se registra el valor de la FCRp de acuerdo al protocolo emitido por la (European Society of Cardiology , 1996), ubicando al sujeto de cúbito dorsal durante cinco (5) minutos; seguido se divide el grupo en dos, para determinar los cadetes que realizan entrenamiento enfocado al G1 respecto al G2 siguiendo el siguiente orden:

1. Se toma la FCRp antes de ejecutar los disparos.

2. Se registra la FC antes y después de ejecutar en la primera fase de diez (10) disparos.

3. Movilidad articular y calentamiento cardiovascular para todo el grupo con intensidad baja hasta 120 ppm, durante quince (15) minutos.

4. El G1 realiza primero tres (3) series de circuito de trabajo de fuerza con autocarga por un (1) minuto de trabajo por uno (1) de descanso, realizando: flexiones de codo, sentadillas y lanzamiento de balón medicinal de cinco (5) kilos. El descanso entre cada serie es de dos (2) minutos, analizando la FC durante la ejecución de los ejercicios. 
5. El G2 realiza primero la carrera continua de 800 metros planos en pista atlética, registrando la FC durante la ejecución.

6. Al finalizar los ejercicios, el G1 y G2 realizan un cambio en sus actividades, pasando a cumplir el protocolo anteriormente descrito; al finalizar estos ejercicios, se procede a desarrollar la segunda fase de disparos manteniendo el protocolo inicial.

7. Finalizando, la sesión se desarrolla una vuelta a la calma por doce (12) minutos.

Se analiza el promedio de efectividad a partir de diez (10) disparos en cada etapa, antes y después del ejercicio aplicado; analizando la efectividad por el número de elementos impactados, según corresponda a la diana. Este elemento, representa la efectividad de 1 a 10, siendo 1 el menor puntaje y 10 el máximo.

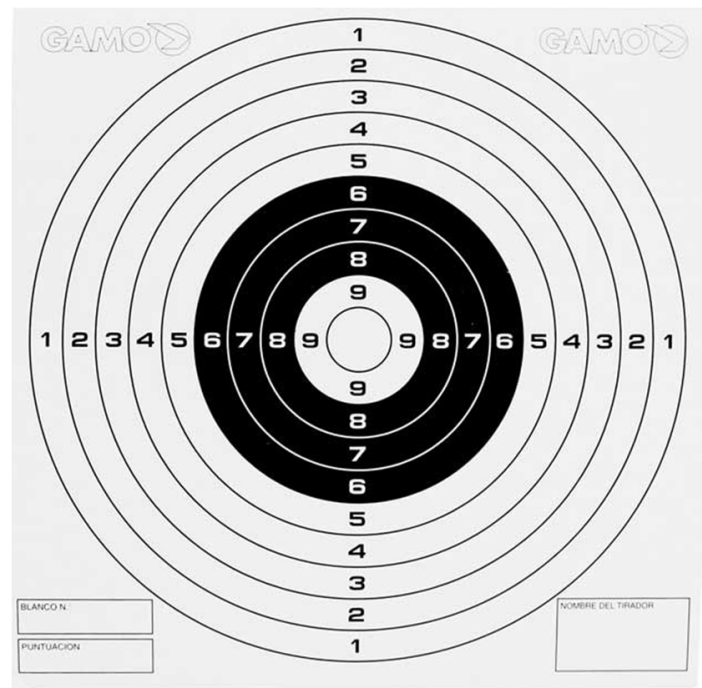

Imagen 1. Diana de cartón para Tiro Olímpico. Tomado de: Real Federación Española de Tiro Olímpico, 2012, p. 57.

\section{RESULTADOS}

Para la comparación entre el comportamiento de la precisión en los disparos antes del entrenamiento y después, así como de la FC durante la sesión, fue usado el Test T-Student para muestras independientes, analizando las varianzas de dos caminos (Two-way Anova), con medidas repetidas para evaluar diferentes momentos de una misma sesión y entre tipos de entrenamiento diferentes.

El análisis de los datos fue realizado con el software Statistical Package for the Social Sciences (SPSS, versión 23.0 SPSS Inc., Chicago IL). Se desarrolla una estadística descriptiva e inferencial para el análisis de los datos. Inicialmente, se desarrolla una parametrización considerando una distribución normal de estos, por medio de la prueba de Shapiro-Wilk a partir de la media aritmética.

La tabla 1. Representa los datos de la población siendo seleccionados diez (10) cadetes al G1 y G2 respectivamente; en cada grupo se encontraban nueve (9) hombres y una (1) mujer. 


\begin{tabular}{lcc}
\hline & $\begin{array}{c}\text { Grupo Fuerza (G1) } \\
\text { Promedio - Desviación }\end{array}$ & $\begin{array}{c}\text { Grupo Resistencia (G2) } \\
\text { Promedio - Desviación }\end{array}$ \\
\hline Edad & $18,45 \pm 4,62$ & $19,03 \pm 2,51$ \\
Peso & $64,99 \pm 8,05$ & $67,39 \pm 7,49$ \\
Talla & $1,71 \pm 0,07$ & $1,69 \pm 0,05$ \\
I M C & $23,44 \pm 2,50$ & $21,96 \pm 2,91$ \\
\hline
\end{tabular}

Tabla 1. Características Sociodemográficas de la muestra

La tabla 2. Representa el comportamiento de la FC en reposo, durante el disparo 1 generando el momento antes del ejercicio aplicado, en la acción del ejercicio que sea de fuerza y/o de resistencia y después de realizar dicha actividad.

\begin{tabular}{lccc}
\hline Variable & $\begin{array}{c}\text { Grupo Fuerza (G1) } \\
\text { Prom }^{1}-\text { Desv. Est }\end{array}$ & $\begin{array}{c}\text { Grupo Resistencia } \\
\text { (G2) } \\
\text { Prom }^{1} \text { - Desv. Est }\end{array}$ & Valor $\mathbf{p}$ \\
\hline FC Reposo & $71 \pm 2,85$ & $69 \pm 3,48$ & No significativo \\
FC antes del disparo 1 & $68 \pm 6,73$ & $71 \pm 5,93$ & No significativo \\
FC después de disparo 1 & $65 \pm 4,31$ & $67 \pm 3,45$ & 0,02 \\
FC antes ejercicio & $75 \pm 3,56$ & $73 \pm 2,51$ & 0,04 \\
FC durante ejercicio & $167 \pm 6,84$ & $145 \pm 7,84$ & 0,01 \\
FC después ejercicio & $146 \pm 4,17$ & $122 \pm 4,39$ & No significativo \\
FC antes del disparo 2 & $127 \pm 5,32$ & $115 \pm 4,91$ & 0,03 \\
FC después de disparo 2 & $95 \pm 10,84$ & $93 \pm 7,21$ & 0,05 \\
\hline
\end{tabular}

Tabla 2. Resultados Frecuencia Cardíaca G1 y G2 - Antes, Durante y Después de invtervención

La tabla 3. Representa el nivel de efectividad analizado desde la diana de disparo en los diez disparos generados antes y después del ejercicio. Se toma el valor promedio por los sujetos de cada grupo. 


\begin{tabular}{lccc}
\hline Variable & $\begin{array}{c}\text { Grupo Fuerza (G1) } \\
\text { Promedio } \\
\text { Desviación }\end{array}$ & $\begin{array}{c}\text { Grupo Resistencia } \\
\text { (G2) } \\
\text { Promedio - } \\
\text { Desviación }\end{array}$ & Valor $\mathbf{p}$ \\
\hline $\begin{array}{l}\text { Efectividad antes del } \\
\text { ejercicio }\end{array}$ & $6,9 \pm 4,15$ & $7,3 \pm 6,15$ & 0,03 \\
$\begin{array}{l}\text { Efectividad después del } \\
\text { ejercicio }\end{array}$ & $5,7 \pm 3,82$ & $8,4 \pm 4,93$ & 0,03 \\
$\quad$ Tabla 3. Efectividad en el dispario pistola $\mathbf{2 0} \mathbf{~ m}$, antes y desoués del ejercicio aplicado
\end{tabular}

\section{DISCUSIÓN}

Inicialmente, se compara el desempeño de los dos grupos de tiradores, sobre la precisión y efectividad en el disparo 41 con el fin de confirmar el efecto del ejercicio generado (fuerza y resistencia) (González-Ravé, Abella, \& Navarro-Valdivieso, 2016). El entrenamiento de la fuerza genera cambios en la dimensión muscular, determinados por la acción muscular generada por la resistencia externa que puede ser la propia masa muscular, pesos libres, bandas elásticas y otros elementos originados por el proceso de la hipertrofia muscular. Así mismo, el entrenamiento de la fuerza debe respetar los contenidos del desarrollo de la intensidad de los ejercicios, la frecuencia de ejecución y el volumen del entrenamiento. Por lo anterior, los métodos de trabajo de alta intensidad se caracterizan por generar cargas a partir de un tiempo determinado, con un número de repeticiones cambiantes y descansos cortos, con variantes en la velocidad de ejecución (Mora, 2015).
De acuerdo a la ejecución y técnica del tiro deportivo es necesario generar una elongación del cuerpo antes de posicionarse para evitar la fatiga del brazo e iniciar un efecto vagal que produzca vibraciones (Neideraver, 2009), esta situación se presenta en el grupo que desarrolla el proceso del entrenamiento de la fuerza, teniendo en cuenta, que la resistencia a la fuerza: "es la capacidad del organismo de soportar la fatiga con rendimientos de la fuerza prolongados" (Weineck, 2012, p. 220), teniendo en cuenta, la intensidad del estímulo, representado en la sesión por el trabajo de entrenamiento de intensidad por la baja densidad (descanso), presentada entre las repeticiones de fuerza. Este trabajo, emite una adaptación en cuanto al potencial de fuerza de las fibras musculares lentas de funcionamiento oxidativo y un mantenimiento a las de contracción rápida (Willmore \& Costill, 2015).

Así mismo, Neideraver (2009), resalta la importancia de la respiración en el momento de generar el disparo, debido a que el ritmo de la frecuencia respiratoria incide en la reducción del ritmo cardíaco para facilitar la concentración y la precisión en el momento 
de disparar; esto se relaciona con el agarre de la empuñadura del arma para ejecutar el tiro, porque no se debe generar una gran presión por parte del puño, sin generar una acción de estrangulamiento; el arma debe ser encajada de modo que la mano quede exactamente en el contorno del puño. El dedo índice debe quedar de modo que el gatillo esté en el medio de la falange distal, sí, el dedo no estuviese en la posición adecuada el tiro puede ser desviado para uno de los lados. De esta forma, la respiración es un factor que debe desarrollar una acción de entrenamiento en el desarrollo de la fuerza, debido a que, se tiene que permitir generar una respiración con el acompañamiento de la presión abdominal, generando una fijación de la caja torácica que es necesaria para llevar la carga y liberar la tensión de forma vertical y evitar el reflejo vagal (Hultman, Spriet, \& Södelund, 2008).

De acuerdo a Weineck (2012), en sujetos entrenados con capacidad cardiorrespiratoria en reposo, la FC está en promedio de 40 a 70 latidos por minuto (l pm), en situación de entrenamiento un sujeto entrenado puede multiplicar su frecuencia cardíaca por cinco, mientras que uno no entrenado la puede triplicar, esto se determina debido a que, en el caso de los sujetos entrenados en el volumen de eyección sistólico superan el doble de la capacidad normal (Billat, 2002). Al inicio de un ejercicio se produce un aumento de la FC, del volumen sistólico y del débito cardíaco, demostrando que la FC y el débito cardíaco empiezan a aumentar en los primeros segundos del ejercicio. De esta forma, de acuerdo a la intensidad del ejercicio entre los 2 a 3 minutos se produce la meseta de la frecuencia cardíaca y el débito, exceptuando situaciones, donde el calor esté por encima de los $35^{\circ}$ y la humedad esté por encima del 55\% (Karvonen, 1983).

En el entrenamiento se presentan intensidades crecientes, por lo cual, la respuesta cardiovascular empieza a aumentar de forma lineal en función a la intensidad del ejercicio (Billat, 2002), por esta situación el entrenamiento de la fuerza genera más cambios y variaciones en la FC por lo que incide, en el control muscular e impide generar una postura controlada en el momento de generar los disparos.

\section{CONCLUSIONES}

El entrenamiento de la fuerza genera un mayor cambio en la FC, por lo que, la postura y la respiración no generan un control adecuado y afecta la precisión en el momento de generar los disparos.

Los trabajos enfocados en el desarrollo de la fuerza por medio del Entrenamiento Intermitente de Alta Intensidad (High Interval Intensity Traininig - HIIT), genera cambios en el Sistema Nervioso Autónomo, reflejando el aumento de las catecolaminas y disparando la FC, alterándola hasta llegar a los valores de Frecuencia Cardíaca Máxima (FCM), la que no disminuyó hasta aplicar trabajos de características aeróbicas.

La aplicación de ejercicios aeróbicos permite disminuir la tensión y aumento del 
volumen de eyección sistólico, controlando la FC de los Cadetes, y permitiendo, desarrollar los disparos de forma controlada y pertinente.
Este estudio se realiza, debido al apoyo de la Facultad de Educación Física Militar y no presenta ningún conflicto de interés por parte de los autores.

\section{REFERENCIAS BIBLIOGRÁFICAS}

FIGUEIREDO, M., \& DE BARROS, J. 2018. Efeitos do condicionamento fisico na frequência cardiaca de repouso e sua variabilidade em indivíduos do gênero masculino sedentàrios e praticantes de exercícicios físicos. Revista Brasileira de Prescrição e Fisiologia do Exercício, pp. 209-220.

CRISTANCHO, H., OTÁLORA, J., \& CALLEJAS, M. 2016. Sistema experto para determinar la frecuencia cardiaca máxima en deportistas con factores de riesgo. Revista Ingeniería Biomédica, pp. 23-31.

FUENTES, J., \& DÍAZA CASASOLA, C. 2010. Analysis of heart rate during a tennis training session and its relationship with heart-healthy index. Journal of Sport and Health Research, pp. 2634.

MANO, C. (2010). O Tiro Esportivo: Um dos elos da corrente da cidadania. Centro de Pesquisas Estratégicas Paulino Soares de Dousa, pp. 1-8.

REAL FEDERACIÓN ESPAÑOLA DE TIRO OLÍMPICO. 2007. Manual de iniciación y adaptación al tiro deportivo. Madrid: Comité Olímpico Español.

MINISTÉRIO DA JUSTIÇA E SEGURANÇA PÚBLICA. 2012. Cartilha de armamento e tiro. Rio de Janiero: Cadernos Informativos Policia Federal.

MINISTERIO DE SALUD Y PROTECCIÓN SOCIAL. 1993. Resolución Número 8430 de 1993. Por la que, se establecen las normas científicas, técnicas y administrativas para la investigación en salud. Bogotá D.C.

EUROPEAN SOCIETY OF CARDIOLOGY. 1996. Standards of measurement, physiological interpretation, and clinical use. European Heart Journal, pp. 354-381.

NEIDERAVER, R. 2009. Tiro Esportivo: Exercício de Concentração. Revista de Villegagnon, pp. 114-117.

WEINECK, J. 2012. Entrenamiento de la fuerza. Em J. Weineck, Entrenamiento Total. Barcelona, España: Editorial Paidotribo, pp. 215-279.

HULTMAN, E., SPRIET, K., \& SÖDELUND, L. (2008). Energy metabolism and fatigue in working muscle. Journal of Sports Sciences, pp. 252-272. 
KARVONEN, J. 1983. Physiological follow-up of endurance runners. Sin Ciudad: Report for the Finnish Sports Foundation.

BILLAT, V. 2002. El sistema cardiovascular: elemento clave del rendimiento deportivo. Em V. Billar, Fisiología y metodología del entrenamiento. Barcelona, España: Editorial Paidotribo, pp. 63-88.

MORA, L. 2015. Fisiología del deporte y el ejercicio. Madrid: Médica Panamericana.

GONZÁLEZ, R., ABELLA, P., \& NAVARRO, M. 2016. Entrenamiento Deportivo. Madrid: Médica Panamericana.

WILLMORE, K., \& COSTILL, E. 2015. Fisiología del deporte y el ejercicio. Madrid, España: Editorial Médica Panamericana.

Cómo citar: Hernández, C., Aroca, J., Herreño, E., \& Gutiérrez, C. (2019). Efectos de una sesión de entrenamiento concurrente sobre la precisión y efectividad en el tiro deportivo con pistola de aire $10 \mathrm{~m}$. R. Actividad fis. y deporte. 6 (1): 58-67.

Artículo de acceso abierto publicado por: Revista Digital: Actividad Física y Deporte, bajo una licencia Creative Commons CC BY-NC 4.0. 